\title{
Structure, function, and localization of aquaporins: their possible implications on gamete cryopreservation
}

\author{
A.D. Sales ${ }^{1}$, C.H. Lobo $^{2}$, A.A. Carvalho ${ }^{1}$, A.A. Moura ${ }^{2}$ and A.P.R. Rodrigues ${ }^{1}$ \\ ${ }^{1}$ Laboratório de Manipulação de Oócitos e Folículos Ovarianos Pré-Antrais, \\ Rede Nordeste de Biotecnologia, Universidade Estadual do Ceará, \\ Fortaleza, CE, Brasil \\ ${ }^{2}$ Grupo de Pesquisa em Biologia da Reprodução, Departamento de Zootecnia, \\ Universidade Federal do Ceará, Fortaleza, CE, Brasil \\ Corresponding author: A.P.R. Rodrigues \\ E-mail: aprrodrigues@gmail.com
}

Genet. Mol. Res. 12 (4): 6718-6732 (2013)

Received April 10, 2013

Accepted October 15, 2013

Published December 13, 2013

DOI http://dx.doi.org/10.4238/2013.December.13.5

\begin{abstract}
The discovery of water channels (aquaporins, AQPs) was a landmark event for the clarification of water transport through the plasma membrane. AQPs belong to a family of intrinsic membrane proteins that act as selective channels for water and for solutes such as glycerol and urea. AQPs were found in different tissues and organs, including male and female reproductive systems. In the swine female reproductive system, the AQPs were localized in the uterus, oviduct, and ovary, as well as in the granulosa cells from primordial follicles. Knowing the involvement of AQPs with the male and female germ cells, as well as their acknowledged role in transporting water through the plasma membrane, the research of these proteins in cryopreservation processes becomes essential. Thus, this review aims to describe the structure and function of AQPs in membranes, highlighting their role
\end{abstract}


in the reproductive system (male and female). We also discuss the involvement of AQPs in cryopreservation, focusing on the effect and importance of these proteins on the rates of vitrification protocols for preantral follicles present in the ovarian tissue of domestic mammals.

Key words: Aquaporins; Cryopreservation; Intracellular ice formation; Preantral follicles; Cryoprotective agents

\section{INTRODUCTION}

The cells of all living organisms are composed mainly of water, a substance with unique features and that is essential for the existence of life on the planet. It is known that water constitutes $95 \%$ of blood plasma, which is responsible for carrying oxygen and nutrients to the cells. Water is the principal constituent of human cells and is involved in all physiological and biochemical processes in an organism. Water allows for the regulation of body temperature, is responsible for toxin removal (through urine and sweat), and is the ideal organic solvent through its hydrogen bonding links. In the extracellular environment, water acts as the main biological component, allowing the passage of solutes into different cells in order to maintain turgor pressure (Lehninger et al., 2005). This water exchange between the intra- and extracellular environments occurs through the plasma membrane (Agre and Kozono, 2003) by simple diffusion, despite the polarity difference. However, there are tissues that require fast water movement (kidneys, for example), where the diffusion is simplified by membrane channels (Lehninger et al., 2005).

The plasma membrane consists of a lipid bilayer, carbohydrates, and proteins (Karp, 2002). Membrane proteins have the ability to recognize and allow the transport of substances into the cell such as ions and sugars, and posteriorly expel those from the cell, thus maintaining a lower cytosolic concentration than the surrounding environment (Chandar and Viselli, 2010). This transport can occur by transmembrane channels formed by proteins called aquaporins (AQPs) (Agre and Kozono, 2003). These proteins pass through the lipid bilayer, allowing the passive water movement from side to side of the plasma membrane, which has a hydrophilic channel that is highly specific to the water molecule. Moreover, AQPs have domains facing both the extracellular and cytoplasmic membrane sides (Karp, 2002), as shown in Figure 1.

The family of AQPs has hundreds of members, expressed in bacteria, plants, and animals of different classes, with different sized channels and substance selectivity. This group of proteins transports small solutes with no ionic charge, such as urea and glycerol (Gomes et al., 2009). Over 10 different types of AQPs have been identified in many mammalian systems, including in the reproductive systems of both males and females (Huang et al., 2006). This is an indication that AQPs play an important role in mammalian reproduction (Thoroddsen et al., 2011).

Besides its importance in cellular physiology, it is believed that AQPs play an important role in the process of cryopreservation of gametes (Edashige et al., 2003). This is based on the assumption that AQPs participate in the transport of water through the membrane, a critical point of cryobiology, because an effective cellular dehydration results in the formation of intracellular ice (Mazur, 1970). However, despite its probable influence on cryopreservation, there are few studies demonstrating the action of these proteins during this process. 


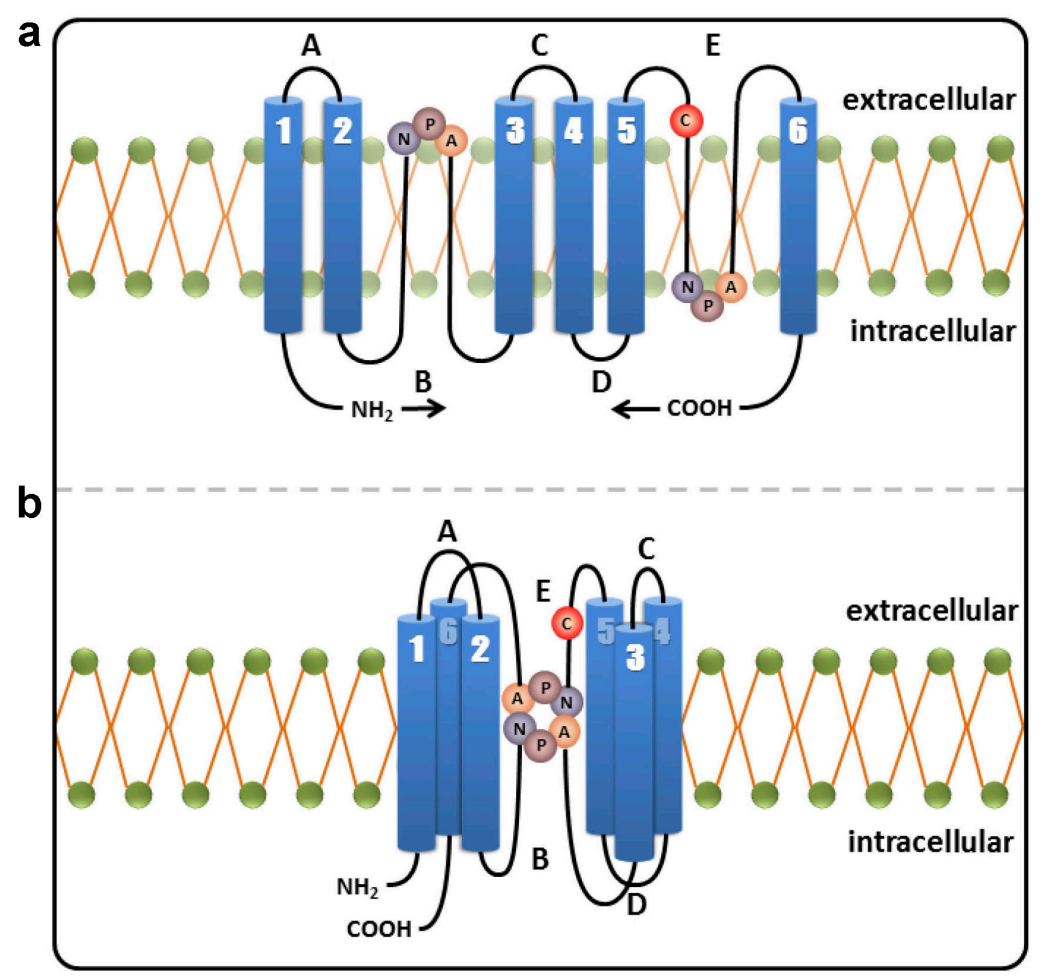

Figure 1. Representation of aquaporin (AQP) structure. a. The structure contains five handle connections (A, B, $\mathrm{C}, \mathrm{D}$, and $\mathrm{E}$ ), where $\mathrm{E}$ and $\mathrm{B}$ contain the conserved motifs NPAs and bend into the molecule forming a narrow portion presenting a hourglass conformation. $\mathbf{b}$. Note that in the region of the channel constriction, the NPA motifs are paired. $\mathrm{C}$, in red, represents the cysteine region where the mercury binds conferring sensitivity in most AQPs. (Adapted from Cheidde and Schorr, 1999).

Therefore, based on the hypothesis of AQP involvement in the cryopreservation process, this review aims to describe the structure and function of these proteins in membranes, highlighting their role in the reproductive system (male and female).

\section{PLASMA MEMBRANE AND WATER TRANSPORT}

The maintenance of biological organisms is related to its ability to maintain osmotic and ionic balance, adapting to alterations in the environment (homeostasis). The body has regulatory mechanisms that maintain extracellular fluid properties, standard chemical concentrations, and appropriate temperature and pressure conditions, so that the cells are capable of performing physiological functions correctly (Agre and Kozono, 2003). Animal cells have a selective membrane that is responsible for defining and separating the intra- and extracellular environments. This membrane is formed by a lipoprotein bilayer with the role of retaining vital components in the cell, such as oxygen for cellular function, and to regulate the entry and exit of substances such as ions and other solutes (Chandar and Viselli, 2010). With few exceptions, the transport of such molecules occurs through transmembrane proteins. These 
proteins are responsible for carrying water molecules, even if these molecules are polar, thereby allowing facilitated diffusion for which a low activation energy $\left(E_{\mathrm{a}}<5 \mathrm{kcal} / \mathrm{mol}\right)$ is used (Fujiyoshi et al., 2002). In addition to transport of molecules, permeability of the membrane to water is also necessary to maintain the osmotic balance. In most cells, water flow occurs freely through the membrane (basal permeability), ensuring the osmotic balance, but in other cells there is a greater control of water flow through the membrane, causing a faster or slower flow rate. The faster flow occurs through proteins called aquaporins (Agre and Kozono, 2003), which are characterized in the next section.

\section{AQUAPORINS}

Aquaporins, also known as AQPs, were first reported in 1988 by Denker et al., who purified a large amount of a $28-\mathrm{kDa}$ protein from human erythrocyte membranes. Grounded with these analyses, the authors proposed AQPs to act as pores or channels that allow water and fluids to flow through the membrane (Denker et al., 1988). By the 1990s, homology was detected among these proteins from several species, including microorganisms and plants, but their molecular function was still unknown. Nevertheless, when the expression of these proteins was induced in Xenopus laevis oocytes, the coefficient of cell water permeability increased approximately 20 times, verifying the role of these proteins as transmembrane water channels; they were then renamed aquaporin-1 (AQP1), but curiously had a lower coefficient compared with that of other aquaporins. The following year, with the help of complementary DNA (cDNA) technology, the peptide sequences of these transmembrane proteins were determined (Preston et al., 1993); at the time, it was believed that these proteins were associated with the high water permeability of erythrocytes.

Ten years later, AQP1 was found in human nephrons, at the proximal renal tubule and the descending portion of Henle's loop (Agre and Kozono, 2003). Subsequent studies have identified more than 10 different types of AQPs in mammals, found in several organs of different species, such as human kidney (Deen et al., 1994), rat epithelial tissue (Lindsay and Murphy, 2007), human blood vessels (Agre and Kozono, 2003), reproductive system of rodents (McConnell et al., 2002), dogs (Domeniconi et al., 2007), and pigs (Skowronski et al., 2009). The studies with AQP1 demonstrated that these proteins are small and integral membrane protein types, crossing the membrane from side to side, and containing two semipores that together form a water channel (Verkman and Mitra, 2000), assisting the transport of solutes (Thoroddsen et al., 2011).

In addition to the transport of these substances, most AQPs in mammals contain extracellular sites susceptible to mercury chloride $\left(\mathrm{HgCl}_{2}\right)$, as well as other metals such as silver nitrate $\left(\mathrm{AgNO}_{3}\right)$ and gold $(\mathrm{Au})$. Such components bind to a cysteine residue, more specifically sulfide groups, inhibiting it, causing a conformational change, and subsequently reducing the water flow (Wan et al., 2004). However, this property is not a general feature for all AQPs; AQP4 and AQP7, for example, have an alanine bound to the cysteine at the position of the $\mathrm{HgCl}_{2}$ binding site, precluding their sensitivity (Cheidde and Schor, 1999).

\section{Structural characterization of aquaporins}

Structurally, AQPs are a class of porins that forms channels, with molecular masses between 28 and $30 \mathrm{kDa}$ (Denker et al., 1988). These proteins have six domains, connected by 
five loops, as shown in Figure 1. In this structure, each polypeptide is formed by a single chain with about 270 amino acids, has terminal amino and carboxyl groups, and is always located in the cell cytoplasm (Preston et al., 1993). AQPs also have three extracellular loops (A, C, and E) and two intracellular loops (B and D) (Fujiyoshi et al., 2002) with the asparagine-proline-alanine sequences fairly conserved, forming short helices and are hydrophobic from opposite sides, associating with substrates selectivity. As shown in Figure 1, most members of this protein family possess a cysteine residue (Cys 189) in the E loop (extracellular), which is situated near the channel responsible for functional sensitivity to mercury, which blocks its function (Preston et al., 1993).

Hydrophilic asparagines are at the center of the channel, each pairing with its homologous group from the opposite loop and forming the pore region (narrowest point of the channel) of about $3 \AA$ (Agre and Kozono, 2003). This pore has electrostatic interactions, where the water molecule, while in Brownian motion (random movement) in the extracellular environment, renders the AQP outer cone walls in a hydrophobic state, causing repulsion (Romeiro et al., 2006). Present in the narrowest region of the cone are two terminal unsaturated amines, which favor hydrogen bonds binding. . Thus, the water dipole binding is aligned with two asparagine molecules and then the molecule crosses the channel (Jensen et al., 2003). This transport is arranged in an iso-osmolar order, at no ATP expense (Jensen et al., 2003).

Some AQPs are also known as aquaglyceroporins (GLPs); the basic difference between these proteins and AQPs is the pore size, a region also known as a selective filter. In some AQPs, the pore measures $\sim 2.8 \AA$, whereas in GLPs, it reaches $\sim 3.4 \AA$. In general, AQPs present a selective region to water (polar region) and contain conserved histidine residues, whereas GLP regions are hydrophobic with two aromatic residues conserved, usually tryptophan and tyrosine. In this sense, the AQP architecture is well preserved, and the specific sidechain pores modulate functional differences (Savage and Stroud, 2007).

\section{Main types and functions of aquaporins}

Based on their permeability, mammalian AQPs are divided into three groups: a) water-selective permeable aquaporins (AQP0, AQP1, AQP2, AQP4, AQP5, AQP6, and AQP8); b) GLPs permeable to water, glycerol, urea, and other solutes (AQP3, AQP7, AQP9, and $\mathrm{AQP10}$ ); c) subcellular aquaporins or super-aquaporins (AQP11 and AQP12), which have low homology with the other AQPs (Murai-Hatano et al., 2008). Aquaporin-0 (AQP0), previously known as the main intrinsic protein, can be found in the lenses of several species; for instance, rodents, cattle, and humans. The lens accommodation in the eye is accompanied by significant volume alterations, which requires the rapid flow of water, with the cell membrane pores ensuring the needed velocity for hydrotransport. Furthermore, AQP0 also seems to act on the local eye microcirculation, since the lens is devoid of blood vessels. The evidence, apart from the involvement of AQP0s in water transport, suggests that this protein also plays a structural role, acting in cell adhesion; that is, gaps in the joints of the fiber lens (Harries et al., 2004). Unlike other aquaporins, the AQP0 permeability to water is remarkably low, around 40 times lower than the inside AQP1 lens (Liu et al., 2011).

AQP1 was the first of these proteins to be characterized in human erythrocytes (Preston et al., 1993). It is expressed mainly in the red blood cells, brain, lungs, and kidneys and is associated with water reabsorption and fluid secretion. Among the identified kidney aquaporins (AQPs 1, 2, 3, 4, and 6), AQP1 is expressed the most, particularly in the proximal tubule 
in the ascending branch, which delivers the route leading to the resorption of water in the proximal nephron (Nielsen et al., 1999). This protein is also expressed in the capillary endothelium, reaffirming its importance for water transport in humans.

AQP2 is expressed mainly in the kidney, and its protein mutation is related with diabetes insipidus (Deen et al., 1994). These mutations are located in the chromosome region $12 q 13$, leading to a deviation or disruption of the function of water channels, affecting both men and women. Studies are thus proving that AQP2 is the only water channel whose function is regulated (Cheidde and Schor, 1999). This same protein was expressed in the endolymphatic sac in the ear in humans, suggesting an active role in the homeostasis of the endolymph (Ishibashi, 2009). AQP2 was also found in the rete testis, efferent ducts, and epididymis of dogs, although its functional role in the reproductive tract is still unknown (Domeniconi et al., 2007).

AQP3 has been found in a wide variety of organs such as the kidneys (Ishibashi et al., 1994), urinary system, digestive system, respiratory system (King et al., 2001), and skin and eyes (Hamann et al., 1998). In most cases, this protein is expressed in epithelial cells or on the lumen surface. Using X. laevis oocytes, several studies showed that this protein carries glycerol and other substances such as ethylene glycol, propylene glycol and acetamide (Yamaji et al., 2006), reasons why it is considered a GLP. Beyond these observations, the permeability and hydraulic conductivity of the channel were evaluated, where it was found that AQP3 plays an important role in transporting ethylene glycol, propylene glycol, and glycerol during bovine embryo cryopreservation.

Regarding AQP4, it is known that this protein is involved with water balance regulation in the brain, since in cases of cerebral edema (ischemic, hypo-osmolar stress-related, tumor-related, traumatic, and inflammatory), there is an increased expression of this protein (King et al., 2001). In humans, an improved expression of AQP4 was confirmed in cases of cerebral ischemia, glial tumors, traumatic brain injury, infection, and inflammatory diseases of the central nervous system (Saadoun et al., 2002). Other authors have proposed that a greater understanding of the molecular channel opens a new dimension for action in the field of brain edema and makes possible the emergence of new therapeutic methods.

As for AQP5, it is mainly expressed in the alveolar epithelium, trachea, and upper bronchus (Gomes et al., 2009). AQP5 has a major task in maintaining water homeostasis in the lungs (Verkman, 2005) and can also regulate the release of saliva in the oral mucosa (Tsubota et al., 2001), and tears and salivary glands (Steinfeld et al., 2001). This protein was also detected in corneal cells, maintaining the epithelium transparency, as well as on its underlying stroma. Interestingly, the expression of AQP5 in the rat uterus increased more rapidly after the administration of estrogen (Ishibashi, 2009).

Regarding AQP6, studies revealed that this protein is present in the membranes of intracellular vesicles, transporting mainly nitrate, and in the renal duct, it demonstrated permeability to some ions such as the chloride ion (Gomes et al., 2009). The special feature of AQP6, proven through transmission electron microscopy, is that this protein is located not on the cell surface but in intracellular vesicles of the renal collecting duct epithelial cells.

AQP7 molecules are involved in water permeability, as well as solutes. This protein is expressed mainly in the adipose tissue, testis, heart, skeletal muscle, and kidneys. In adipose tissue, the presence of AQP7 seems to be tied to a greater ease of glycerol secretion (Rodriguez et al., 2006). AQP7 also transports nonionic solute surfactants with low molecular weight. In follicular maturation, studies have confirmed the presence of this protein in granulosa cells, and it is believed to be related with antrum formation (Huang et al., 2006). 
AQP8 is expressed in many organs and tissues, especially the pancreas, liver, salivary glands, kidneys, testes, epididymis, duodenum, stomach, jejunum, lung, trachea, and placenta (Liu et al., 2006). The induction of this recombinant protein in rats, mice, and humans demonstrated its action on carrying water; additionally, in rats and humans, AQP8s are not permeable to urea and glycerol. Furthermore, in humans, AQP8 is permeable to ammonia analogs (formamide and methylammonium), suggesting that this AQP can carry in vivo ammonia, contributing physiologically to the acid-base balance (Liu et al., 2006).

AQP9 has been identified in the liver, leukocytes, testis, brain, and ovary (Gomes et al., 2009) and is involved in the permeability of water and small solutes. Its role in the brain is still unknown; however, its permeability to lactate and glycerol indicates that this protein may be important in energy metabolism (Tait et al., 2008). AQP9 was also localized in granulosa cells from developing follicles in the oviduct and uterus of sows (Skowronski et al., 2009).

AQP10 was found in the small intestine of humans, particularly in the jejunum (Ishibashi et al., 2002), presenting the same function as GLPs, and is also present in absorptive epithelial cells. Interestingly, a study in mice revealed that AQP10 is a pseudogene. In an attempt to elucidate the physiological significance of this protein, the authors have cloned the AQP10 gene and the results showed that this gene was not detected in the jejunum or, indeed, any other tissue of that species. Additionally, it was revealed that the gene in charge of AQP10 expression in mice contains several mutations, which leads to production of a non-functional protein. Another feature of this protein, in humans, is that their absorption is inhibited in the presence of glycerol derivatives, such as ethylene glycol and propylenediol (Ishii et al., 2011). However, this mechanism is not yet fully elucidated, requiring further research.

AQP11 and AQP12 belong to a new aquaporin subfamily called super-aquaporins (Ishibashi, 2009). AQP11 is expressed in the kidneys, brain, liver, testes, heart, intestine, and adipose tissue (Gorelick et al., 2006), but its function is still unknown (Buffoli, 2010). Interestingly, when AQP11 was expressed in X. laevis oocytes, there was no water transportation (Gorelick et al., 2006). On the other hand, when the protein was reconstituted into liposomes, a channel of high activity to water was evident. Currently, the reason for this discrepancy is not yet clear, requiring further study to understand AQP11's function within the cell. AQP12 is found only in pancreatic acinar cells (Buffoli, 2010). The specific location has not been established for this subfamily, but its presence in the pancreas implies a possible pathway in digestive enzyme secretion and pancreatic cell fluids (Rojek et al., 2008). AQP12 has a higher identity with AQP11 (32\%) compared with other AQPs, which show less than $15 \%$ identity (Ishibashi, 2009). A structural feature of AQP12 is the lack of an N-terminal cytoplasmic region, in contrast to all other known aquaporins (Ishibashi, 2009). Table 1 shows a summary of the location of all AQPs found in various mammalian species, which were based on polymerase chain reaction, Northern blot, or microarray results, and have not necessarily been confirmed by immunohistochemistry.

\section{AQUAPORINS AND THE REPRODUCTIVE SYSTEM}

As described above, the AQPs can be found in many mammalian systems. In particular, AQPs have been found in various organs of the reproductive system, regardless of sex (Jablonski et al., 2003), thereby demonstrating its function in mammalian reproduction. 


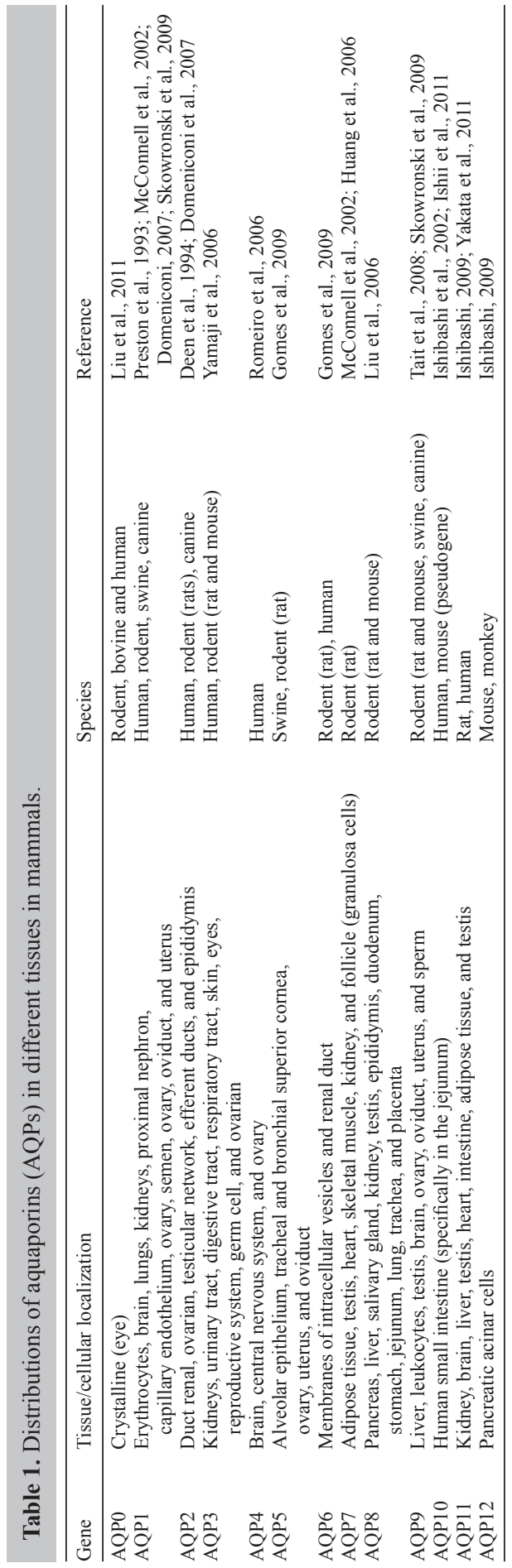




\section{Male reproductive system}

AQPs are found in the intra- and extratesticular spermatic pathways (Domeniconi et al., 2007). In the male reproductive system, several AQPs have been expressed in different locations, such as the testes (AQP0, AQP1, AQP7, AQP8, and AQP9), efferent ducts (AQP1, AQP9, and AQP10), epididymis (AQP1, AQP3, AQP9, and AQP10), vas deferens (AQP1, AQP2, and AQP9), and accessory glands (AQP1 and AQP9) of adult mammals (Huang et al., 2006). In addition to these, AQPs 3 and 10 have also been described in rats (Hermo et al., 2004). Recent studies detected two new AQPs in mammals, AQPs 11 and 12, with AQP11 being present in the testes and kidney, whereas AQP12 is selectively expressed in the pancreas (Ishibashi, 2009).

Among the GLPs, AQP3 is located in the epididymal basal cells (Hermo et al., 2004). AQP7 is located throughout the tail of the sperm, except at the end, as reported in humans. The expression of this protein was also found in germ cells (spermatids) of adults and developing rats (Huang et al., 2006), and in testicular and epididymal spermatozoa (Calamita et al., 2001), indicating a possible role in storage of sperm maturation as well as in late spermatogenesis. However, in non-sterile knockout mice, semen analysis demonstrated no morphological or functional abnormalities, suggesting that this AQP is turned on and off at different times (Sohara et al., 2009).

In rats and mice, AQP9 was expressed in immature germ cells (Tsukaguchi et al., 1998), efferent ducts, and over the epididymis and vas deferens of dogs, showing that this protein is abundantly expressed in the testis (Domeniconi et al., 2007). In the epididymal cells, AQP9 was expressed in greater quantities in the initial and caudal segments of the epididymis (Badran and Hermo, 2002). These AQPs as well as AQP1, present in the efferent duct and epididymis, may be involved in fluid absorption, promoting sperm concentration, suggesting for this epithelium a great potential related to the movement of water and small solutes along the epididymis (Hermo and Smith, 2011).

On the other hand, AQP10 was found in the epithelium of efferent ducts (Hermo et al., 2004). In the literature, there are no studies associating AQPs with fertility or infertility in men. However, these studies may be the key to uncovering the interaction of these proteins to the maintenance of the germinal epithelium, the powerful functionality of sperm production, and natural fertilization.

\section{Female reproductive system}

The first reports of the presence of AQPs in the female reproductive system occurred in the human uterus, after cDNA isolation and sequencing. These authors presented that the cloned cDNA had $99.8 \%$ homology to the AQP1 found in human erythrocytes. Among the AQPs found, nine isoforms have been expressed in the female reproductive system of humans, rats, and mice (Huang et al., 2006). Studies have demonstrated the expression of AQPs in the uterus of rats (AQPs 5 and 9, Lindsay and Murphy, 2007), mice (AQPs 0, 1, 4, and 5, Richard et al., 2003; AQPs 1, 3, and 8, Jablonski et al., 2003), pigs (AQPs 1, 5, and 9, Skowronski et al., 2009), and humans (AQPs 1-4, Thoroddsen et al., 2011). AQP1 expression was detected in the rat uterus, suggesting that these proteins represent a control system for water movement across the intraluminal, interstitial, and capillary compartments (Skowronski et al., 2009). 
In the oviduct of female rats, the presence of AQPs 5, 8, and 9 was observed only in the epithelial cells. In addition, studies found that AQP9 was the only AQP localized after ovariectomy and administration of progesterone and estradiol (Branes et al., 2005). These results indicate that AQPs function not only in water transport but also in the absorption or secretion of luminal fluid metabolites, thus maintaining a suitable environment for reproductive functions (Kenny et al., 2002). During the estrous cycle of rats, a variation of AQP9 was observed, with higher levels developing during the proestrus and estrus phases. This finding indicates that steroid hormones can regulate the expression of this protein and thus control water transport into the oviduct lumen (Branes et al., 2005).

In a study conducted by Skowronski et al. (2009), only three of the AQPs (1, 5, and 9) were expressed in the female reproductive organs. AQP1 was localized in the ovary, oviduct, and uterus. AQP5, besides being present in these organs, was also localized in granulosa cells of primordial follicles, smooth muscle and luminal cells, and glandular epithelial cells. AQP9 was already observed in the granulosa cells of developing follicles, in the oviduct and uterus, and in ovarian epithelial cells. This study also suggested that the ovarian AQP9 does not control water transport only. The strong expression of this protein in ovarian tissues could have an important implication in follicular development, because it is a GLP and therefore allows the passage of other electrolytes. In this case, the protein could ensure a sufficient supply of estrogen to granulosa cells, making it essential for follicle development (Skowronski et al., 2009).

According to the literature, it is assumed that sex hormones may directly regulate the transcription of AQP genes, but the effect may be mediated through an indirect route that involves the expression of other intracellular mediators. The permeability regulators that vary along the reproductive cycle, such as the transcription from receptors to the vascular endothelial growth factor, and the receptor for the platelet activation factor expressed by epithelial cells of the uterine tube, are possible explanations for this mechanism (Jablonski et al., 2003). There is evidence that AQPs may participate in the regulation of steroid hormones produced by ovarian follicles (Jablonski et al., 2003; Huang et al., 2006). However, the limited results about AQPs in the ovary suggest that its main function in this organ is in antrum formation and expansion (Thoroddsen et al., 2011).

The exact mechanism for the fluid transport from layer to layer of teak granulosa cells and the formation of avascular follicular fluid is not yet completely understood (Rodgers and Irving-Rodgers, 2010), but it is a slow process and not likely to occur by simple diffusion. Studies indicate that the AQPs present in these follicles were responsible for the rapid passage of a large volume of water into the antral cavity (Preston et al., 1993).

A study of the human ovary verified AQPs (1-4) expressions at different times of the preovulatory follicle, in the theca and granulosa cells, and observed their expressions in the follicles during ovulation. In this same study, there was a sharp increase in AQPs 2 and 3 at the beginning of ovulation, suggesting a role in follicular rupture, and a high expression of AQP1 at the end of this event, indicating its function in corpus luteum formation. Thus, it is believed that the AQPs also exert a critical key role in ovulation (Thoroddsen et al., 2011). In Figure 2, we can see the presence of this protein at different times of folliculogenesis.

\section{IMPORTANCE OF AQUAPORINS TO CRYOPRESERVATION OF MALE AND FEMALE GAMETES}

In the last decade, the advances in understanding about the cryobiology phenomenon, 
which aims at preserving biological material for an undetermined time in low temperatures (Mazur, 1970), contributed to the development of accurate methods for the preservation of cells, mainly related to gametes. The cryopreservation of mammalian gametes and embryos has been used successfully in many aspects, including the preservation of the genetic variability in laboratory animals, the reproduction of production animals, and assisted reproduction in human beings (Rall, 2001).
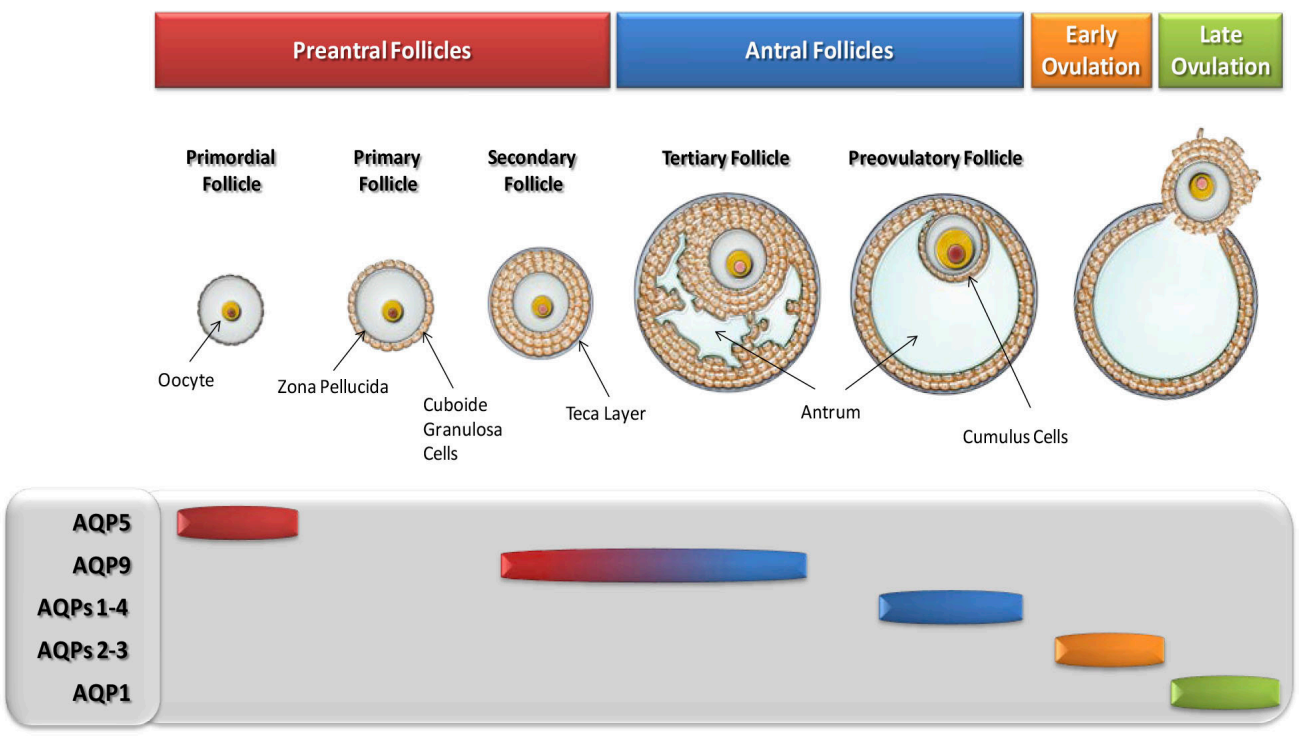

Figure 2. Attendance of aquaporins (AQPs) in folliculogenesis. AQP5: in granulosa cells in porcine (Skowronski et al., 2009), AQP9: in granulosa cells (Skowronski et al., 2009), AQPs 1-4: ovulation period, AQP1: final ovulation (Thoroddsen et al., 2011).

The process of cryopreservation can be achieved by using two methods: slow freezing and vitrification. With regard to slow freezing, this is based on cell dehydration, the association of a soft osmotic balance, the use of solutions that contain low concentrations of cryoprotectant agents (CPAs), and a gradual reduction of temperature, usually by using a programmable freezer. Vitrification involves a fast substitution of the intracellular water with solutions containing high concentrations of CPA, and an abrupt reduction of temperature, aiming for the formation of a vitreous state without the formation of ice crystals. Regardless of the method of cryopreservation used, the addition of CPAs is indispensable to providing cell cryoprotection, allowing the cells to be able to hold up to the injuries inherent in the procedures of cryopreservation (Santos, 2005). However, cell cryopreservation in large amounts and of multicellular systems still continues to be a challenge in some cases, owing to intracellular ice formation (IIF), the toxicity of the CPAs, and the risks of osmotic shock, especially at the moment of thawing and removal of the cryoprotectant agent (Mazur, 1984).

Specifically, in relation to IIF, it is hypothesized that these crystals may be able to form and grow themselves through the canals (AQPs) formed in the membrane, although there is evidence that the ice formed has a larger size in comparison with these canals (Mazur, 
1970). In the process of cryopreservation, there are two moments that are crucial to the IIF. One of these is when the CPA is added (balance period), especially in the slow freezing process, wherein there has to be a way for the water to exit (cellular dehydration) concurrently with the CPA entrance in order to avoid the formation of new crystals or even the increase of small crystals inside of the cell or the tissue. Another moment is the thawing, which must take place quickly in order to avoid the formation of new crystals or even the growth of small crystals previously formed (Mazur, 1970). Thus, taking into consideration the IIF hypothesis, and based on the need for maximum removal of the intracellular water before the process of cryopreservation, especially in the process of slow freezing, associated with the fact that the AQPs accelerate the transportation of water in the cell, it is acknowledged that these proteins perform a role in the transportation of water, which is extremely important in the process of cellular cryopreservation.

As described in the sections above, some AQPs are permeable to glycerol and urea, as well as to metalloids and a large variety of non-charged solutes (carbamide, polyols, purines, pyrimidine), including the CPAs, such as propyleneglycol, ethyleneglycol, acetamide, and, possibly, dimethyl sulfoxide (Chauvigné et al., 2011). This factor is extremely important, considering that during the balance period or during CPA removal, there must be a perfect control of the water and CPA flow through the cellular membrane; that is, the water leaving and the CPA entering in the first case, and the water entering and CPA leaving in the second case.

A study using mice demonstrated the expression of AQP3 in mature oocytes (metaphase II) and in embryos at various stages of development. With regard to the cryopreservation of these structures, the authors reported that this protein performs a significant role in the transportation of water, as demonstrated by the use of artificial AQP3, which increased the permeability to water as well as to glycerol. The result of this effect was the increase of oocyte surviving after vitrification. In addition, in this study, the hydraulic conductivity and the glycerol permeability were also evaluated, confirming that the formulation of the artificial AQP3 improved the permeability of the cryoprotectant agent (Edashige et al., 2003).

The studies involving these proteins in the cryopreservation of mammals are still scarce and in the initial stages. Thus, it is extremely important to conduct new studies to assess the effects of AQPs in this process, with the aim to perfect this technique and consequently to increase the success rates of cryopreservation of embryos and gametes.

\section{FINAL CONSIDERATIONS}

As discussed, the AQPs are proteins existing in different mammalian tissues and that have the main function of water transportation, although they can perform other specific functions. In the reproduction system, for example, they can be in various locations independently of gender. In males, AQPs are related to the motility, maturation, and storage capacity of sperm. In the case of the female system, AQPs play important roles in follicle development and the maintenance of female fertility. Knowing the involvement of AQPs in the female and male germ cells, as well as their recognized role in water transportation across the plasma membrane, it is essential to investigate this family of proteins in the process of cryopreservation of these structures.

Despite the fact that gene analysis and expression are essential for the understanding of tissue physiology, there are no mammalian studies being conducted to examine the gene 
expression of proteins involved in the important processes for survival after cryopreservation. In the survival process, the exposure of cells to the extreme cold can result in irreparable injury caused by the formation of intracellular ice crystals, which must be avoided or minimized. It is hypothesized that during the formation of intracellular ice, external ice makes contact with the ice surface on the cells and grows through preexisting pores in the membrane of the cell to the interior of the nucleated cell, a process caused by the presence of the AQPs (Mazur, 1984). In attempts to solve this problem, CPAs are used but they also increase the risk of cellular toxicity. However, regardless of how crystals of ice are formed in the interior of cells, the formation of these crystals must be avoided through removal of the maximum possible quantity of intracellular water (Faustino et al., 2011). Therefore, whether or not aquaporin expression is involved in the efficiency of this process must be assessed, and consequently a positive effect will be made in relation to the viability of gametes after cryopreservation, contributing in a significant way to the perfection of cryopreservation protocols.

\section{ACKNOWLEDGMENTS}

Research supported by Fundação Cearense de Apoio ao Desenvolvimento Científico e Tecnológico - FUNCAP (Ceará State Research Council), Coordenação de Aperfeiçoamento de Pessoal de Nível Superior - CAPES (Brazilian Ministry of Education), and Conselho Nacional de Pesquisa e Desenvolvimento - CNPq (Brazilian National Research Council).

\section{REFERENCES}

Agre P and Kozono D (2003). Aquaporin water channels: molecular mechanisms for human diseases. FEBS Lett. 555: $72-78$.

Badran HH and Hermo LS (2002). Expression and regulation of aquaporins 1, 8, and 9 in the testis, efferent ducts, and epididymis of adult rats and during postnatal development. J. Androl. 23: 358-373.

Branes MC, Morales B, Rios M and Villalon MJ (2005). Regulation of the immunoexpression of aquaporin 9 by ovarian hormones in the rat oviductal epithelium. Am. J. Physiol. Cell Physiol. 288: C1048-C1057.

Buffoli B (2010). Aquaporin biology and nervous system. Curr. Neuropharmacol. 8: 97-104.

Calamita G, Mazzone A, Bizzoca A and Svelto M (2001). Possible involvement of aquaporin-7 and -8 in rat testis development and spermatogenesis. Biochem. Biophys. Res. Commun. 288: 619-625.

Chandar N and Viselli S (2010). Cell and Molecular Biology (Lippincott's illustrated reviews series). International edn. Lippincott Williams \& Wilkins, Philadelphia.

Chauvigné F, Zapater C and Cerda J (2011). Role of aquaporins during teleost gametogenesis and early Embryogenesis. Front. Physiol. 2: 66.

Cheidde L and Schor N (1999). Review: water transporters. Rev. Assoc. Med. Bras. 45: 71-78.

Deen PM, Verdijk MA, Knoers NV, Wieringa B, et al. (1994). Requirement of human renal water channel aquaporin-2 for vasopressin-dependent concentration of urine. Science 264: 92-95.

Denker BM, Smith BL, Kuhajda FP and Agre P (1988). Identification, purification, and partial characterization of a novel Mr 28,000 integral membrane protein from erythrocytes and renal tubules. J. Biol. Chem. 263: 15634-15642.

Domeniconi RF, Orsi AM, Justulin LA Jr, Beu CC, et al. (2007). Aquaporin 9 (AQP9) localization in the adult dog testis excurrent ducts by immunohistochemistry. Anat. Rec. 290: 1519-1525.

Edashige K, Yamaji Y, Kleinhans FW and Kasai M (2003). Artificial expression of aquaporin-3 improves the survival of mouse oocytes after cryopreservation. Biol. Reprod. 68: 87-94.

Faustino LR, Silva CMG, Rossetto R and Rodrigues GQ (2011). Estado atual e desafios da criopreservação de tecido ovariano em mamíferos. Rev. Bras. Reprod. Anim. 35: 3-15.

Fujiyoshi Y, Mitsuoka K, de Groot BL, Philippsen A, et al. (2002). Structure and function of water channels. Curr. Opin. Struct. Biol. 12: 509-515.

Gomes D, Agasse A, Thiebaud P, Delrot S, et al. (2009). Aquaporins are multifunctional water and solute transporters 
highly divergent in living organisms. Biochim. Biophys. Acta 1788: 1213-1228.

Gorelick DA, Praetorius J, Tsunenari T, Nielsen S, et al. (2006). Aquaporin-11: a channel protein lacking apparent transport function expressed in brain. BMC Biochem. 7: 14.

Hamann S, Zeuthen T, la Cour M, Nagelhus EA, et al. (1998). Aquaporins in complex tissues: distribution of aquaporins 1-5 in human and rat eye. Am. J. Physiol. 274: C1332-C1345.

Harries WE, Akhavan D, Miercke LJ, Khademi S, et al. (2004). The channel architecture of aquaporin 0 at a 2.2-A resolution. Proc. Natl. Acad. Sci. U. S. A. 101: 14045-14050.

Hermo L and Smith CE (2011). Thirsty business: cell, region, and membrane specificity of aquaporins in the testis, efferent ducts, and epididymis and factors regulating their expression. J. Androl. 32: 565-575.

Hermo L, Krzeczunowicz D and Ruz R (2004). Cell specificity of aquaporins 0, 3, and 10 expressed in the testis, efferent ducts, and epididymis of adult rats. J. Androl. 25: 494-505.

Huang HF, He RH, Sun CC, Zhang Y, et al. (2006). Function of aquaporins in female and male reproductive systems. Hum. Reprod. Update 12: 785-795.

Ishibashi K (2009). New members of mammalian aquaporins: AQP10-AQP12. Handb. Exp. Pharmacol. 251-262.

Ishibashi K, Sasaki S, Fushimi K, Uchida S, et al. (1994). Molecular cloning and expression of a member of the aquaporin family with permeability to glycerol and urea in addition to water expressed at the basolateral membrane of kidney collecting duct cells. Proc. Natl. Acad. Sci. U. S. A. 91: 6269-6273.

Ishibashi K, Morinaga T, Kuwahara M, Sasaki S, et al. (2002). Cloning and identification of a new member of water channel (AQP10) as an aquaglyceroporin. Biochim. Biophys. Acta 1576: 335-340.

Ishii M, Ohta K, Katano T, Urano K, et al. (2011). Dual functional characteristic of human aquaporin 10 for solute transport. Cell. Physiol. Biochem. 27: 749-756.

Jablonski EM, McConnell NA, Hughes FM Jr and Huet-Hudson YM (2003). Estrogen regulation of aquaporins in the mouse uterus: potential roles in uterine water movement. Biol. Reprod. 69: 1481-1487.

Jensen MO, Tajkhorshid E and Schulten K (2003). Electrostatic tuning of permeation and selectivity in aquaporin water channels. Biophys. J. 85: 2884-2899.

Karp G (2002). Cell and Molecular Biology. 3rd edn. John Wiley \& Sons, Inc., New York.

Kenny DA, Humpherson PG, Leese HJ, Morris DG, et al. (2002). Effect of elevated systemic concentrations of ammonia and urea on the metabolite and ionic composition of oviductal fluid in cattle. Biol. Reprod. 66: 1797-1804.

King LS, Choi M, Fernandez PC, Cartron JP, et al. (2001). Defective urinary-concentrating ability due to a complete deficiency of aquaporin-1. N. Engl. J. Med. 345: 175-179.

Lehninger AL, Nelson DL and Cox MM (2005). Principles of Biochemistry. 4th edn. W.H. Freeman \& Co, New York.

Lindsay LA and Murphy CR (2007). Aquaporins are upregulated in glandular epithelium at the time of implantation in the rat. J. Mol. Histol. 38: 87-95.

Liu J, Xu J, Gu S, Nicholson BJ, et al. (2011). Aquaporin 0 enhances gap junction coupling via its cell adhesion function and interaction with connexin 50. J. Cell Sci. 124: 198-206.

Liu K, Nagase H, Huang CG, Calamita G, et al. (2006). Purification and functional characterization of aquaporin-8. Biol. Cell 98: 153-161.

Mazur P (1970). Cryobiology: the freezing of biological systems. Science 168: 939-949.

Mazur P (1984). Freezing of living cells: mechanisms and implications. Am. J. Physiol. 247: C125-C142.

McConnell NA, Yunus RS, Gross SA, Bost KL, et al. (2002). Water permeability of an ovarian antral follicle is predominantly transcellular and mediated by aquaporins. Endocrinology 143: 2905-2912.

Murai-Hatano M, Kuwagata T, Sakurai J, Nonami H, et al. (2008). Effect of low root temperature on hydraulic conductivity of rice plants and the possible role of aquaporins. Plant Cell Physiol. 49: 1294-1305.

Nielsen S, Kwon TH, Christensen BM, Promeneur D, et al. (1999). Physiology and pathophysiology of renal aquaporins. J. Am. Soc. Nephrol. 10: 647-663.

Preston GM, Jung JS, Guggino WB and Agre P (1993). The mercury-sensitive residue at cysteine 189 in the CHIP28 water channel. J. Biol. Chem. 268: 17-20.

Rall WF (2001). Cryopreservation of Mammalian Embryos, Gametes, and Ovarian Tissues: Current Issues and Progress. In: Assisted Fertilization and Nuclear Transfer in Mammals (Wolf DP and Zelinski-Wooten MB, eds.). 1st edn. Humana Press Inc., Totowa, 173-187.

Richard C, Gao J, Brown N and Reese J (2003). Aquaporin water channel genes are differentially expressed and regulated by ovarian steroids during the periimplantation period in the mouse. Endocrinology 144: 1533-1541.

Rodgers RJ and Irving-Rodgers HF (2010). Formation of the ovarian follicular antrum and follicular fluid. Biol. Reprod. 82: 1021-1029.

Rodriguez A, Catalan V, Gomez-Ambrosi J and Fruhbeck G (2006). Role of aquaporin-7 in the pathophysiological control of fat accumulation in mice. FEBS Lett. 580: 4771-4776. 
Rojek A, Praetorius J, Frokiaer J, Nielsen S, et al. (2008). A current view of the mammalian aquaglyceroporins. Annu. Rev. Physiol. 70: 301-327.

Romeiro RR, Teixeira AL, Silva RMA, De Marco La, et al. (2006). O papel da aquaporina-4 no edema cerebral. Rev. Neucienc. 14: 220-224.

Saadoun S, Papadopoulos MC, Davies DC, Krishna S, et al. (2002). Aquaporin-4 expression is increased in oedematous human brain tumours. J. Neurol. Neurosurg. Psychiatr. 72: 262-265.

Santos RR (2005). Criopreservação de Folículos Pré-Antrais Ovinos. Doctoral thesis, Faculdade de Medicina Veterinária, UECE, Fortaleza.

Savage DF and Stroud RM (2007). Structural basis of aquaporin inhibition by mercury. J. Mol. Biol. 368: 607-617.

Skowronski MT, Kwon TH and Nielsen S (2009). Immunolocalization of aquaporin 1, 5, and 9 in the female pig reproductive system. J. Histochem. Cytochem. 57: 61-67.

Sohara E, Uchida S and Sasaki S (2009). Function of aquaporin-7 in the kidney and the male reproductive system. Handb. Exp. Pharmacol. 219-231.

Steinfeld S, Cogan E, King LS, Agre P, et al. (2001). Abnormal distribution of aquaporin-5 water channel protein in salivary glands from Sjogren's syndrome patients. Lab. Invest. 81: 143-148.

Tait MJ, Saadoun S, Bell BA and Papadopoulos MC (2008). Water movements in the brain: role of aquaporins. Trends Neurosci. 31: 37-43.

Thoroddsen A, Dahm-Kahler P, Lind AK, Weijdegard B, et al. (2011). The water permeability channels aquaporins 1-4 are differentially expressed in granulosa and theca cells of the preovulatory follicle during precise stages of human ovulation. J. Clin. Endocrinol. Metab. 96: 1021-1028.

Tsubota K, Hirai S, King LS, Agre P, et al. (2001). Defective cellular trafficking of lacrimal gland aquaporin-5 in Sjogren's syndrome. Lancet 357: 688-689.

Tsukaguchi H, Shayakul C, Berger UV, Mackenzie B, et al. (1998). Molecular characterization of a broad selectivity neutral solute channel. J. Biol. Chem. 273: 24737-24743.

Verkman AS (2005). More than just water channels: unexpected cellular roles of aquaporins. J. Cell Sci. 118: 3225-3232.

Verkman AS and Mitra AK (2000). Structure and function of aquaporin water channels. Am. J. Physiol. Renal Physiol. 278: F13-F28.

Wan X, Steudle E and Hartung W (2004). Gating of water channels (aquaporins) in cortical cells of young corn roots by mechanical stimuli (pressure pulses): effects of $\mathrm{ABA}$ and of $\mathrm{HgCl}_{2}$. J. Exp. Bot. 55: 411-422.

Yakata K, Tani K and Fujiyoshi Y (2011). Water permeability and characterization of aquaporin-11. J. Struct. Biol. 174: 315-320.

Yamaji Y, Valdez DM Jr, Seki S, Yazawa K, et al. (2006). Cryoprotectant permeability of aquaporin-3 expressed in Xenopus oocytes. Cryobiology 53: 258-267. 